\title{
Utilização de sal mineral rico em molibdênio na prevenção da intoxicação cúprica acumulativa em ovinos - microminerais hepáticos
}

\author{
[Use of molybdenum rich mineral mix in the prevention of cumulative cooper \\ poisoning in sheep - hepatic microminerals] \\ A.C. Antonelli ${ }^{1}$, R.A. Barrêto Júnior ${ }^{2}$, C.S. Mori ${ }^{3}$, A.H.H. Minervino ${ }^{4}$, U.M. Schalch ${ }^{5}$, \\ J.C.G. Pacheco ${ }^{5}$, E.L. Ortolani ${ }^{3}$ \\ ${ }^{1}$ Universidade Federal do Vale do São Francisco - Univasf - Petrolina, PE \\ ${ }^{2}$ Universidade Federal Rural do Semi-Árido - Ufersa - Mossoró, RN \\ ${ }^{3}$ Faculdade de Medicina Veterinária e Zootecnia - Universidade de São Paulo - FMVZ-USP - São Paulo, SP \\ ${ }^{4}$ Universidade Federal do Oeste do Pará - Ufopa - Santarém, PA \\ ${ }^{5}$ Faculdade de Medicina Veterinária e Zootecnia - Universidade de São Paulo - FMVZ-USP - Pirassununga, SP
}

\begin{abstract}
RESUMO
Este estudo avaliou a capacidade de um sal mineral rico em molibdênio (Mo) em prevenir a intoxicação cúprica acumulativa (ICA), mediante a análise das variáveis clínicas e dos teores de cobre $(\mathrm{Cu})$ e $\mathrm{Mo}$ hepático. Foram utilizados 25 ovinos cruzados da raça Ile-de-France, aleatoriamente distribuídos igualmente em cinco grupos, em que o grupo 1 recebia dieta contendo $80 \%$ de volumoso e $20 \%$ de concentrado, os grupos 2 e 3 recebiam $50 \%$ de volumoso e $50 \%$ de concentrado, e os grupos 4 e 5 recebiam a mesma dieta dos grupos 2 e 3, com a adição diária de $150 \mathrm{mg}$ de sulfato de $\mathrm{Cu}$. Os grupos 1, 3 e 5 recebiam sal mineral contendo 300ppm de Mo. Foram realizadas três biópsias hepáticas para determinação de $\mathrm{Cu}$, Mo e Zn. Três ovinos do grupo 4 e um do grupo 5 manifestaram ICA. Não houve diferença na frequência de mortalidade entre os grupos $(\mathrm{P}=0,56)$. Os teores de $\mathrm{Cu}$ hepático nos ovinos com ICA (2450ppm) foram superiores aos que não intoxicaram (1518ppm). Quanto maior a ingestão de Mo na dieta, menor foi o acúmulo de $\mathrm{Cu}$ hepático ao término do experimento $(\mathrm{r}=-0,72)$.
\end{abstract}

Palavras-chave: carneiro, cobre, antagonistas, fígado

\begin{abstract}
The aim of this project is to evaluate the capacity of a molybdenum-rich mineral salt in the prevention of cumulative cooper poisoning (CCP) in sheep, through clinical and hepatic copper and molybdenum concentrations. Twenty five crossbreed Ile-de-France sheep were randomly distributed equally into five groups. Group 1 received a $80 \%$ forage and $20 \%$ concentrate diet, groups 2 and 3 received a 50\% forage and 50\% concentrate diet, and groups 4 and 5 received the same diet as groups 2 and 3 with a daily supplementation of $150 \mathrm{mg}$ of copper sulfate. Groups 1, 3 and 5 received a mineral salt with 300 ppm of molybdenum. Three times during the experiment a liver biopsy was carried out to evaluate the degree of copper accumulation. Three sheep from group 4 and one sheep from group 5 showed a clinical picture of $C C P$. There was no difference in the frequency of mortality between groups 4 and $5(P=0.56)$. The liver copper concentration was higher in sheep with CCP (2450 ppm) compared to sheep that did not present CCP (1518 ppm). The higher the ingestion of molybdenum in the diet the lower the liver copper concentration at the end of the experiment $(r=-0.72)$.
\end{abstract}

Keywords: lamb, copper, antagonists, lamb, liver

Recebido em 9 de setembro de 2015

Aceito em 15 de novembro de 2015

E-mail: alexandre.antonelli@univasf.edu.br 


\section{INTRODUÇÃO}

O excesso ou a falta de cobre, semelhantemente ao que ocorre com outros macro e microelementos, pode provocar tanto a intoxicação quanto a carência em animais, respectivamente (Suttle, 2010). Entre as espécies domésticas, sem dúvida a ovina é a mais predisposta a desenvolver o quadro carencial e a intoxicação. Esses quadros estão ligados a diferenças marcantes no metabolismo do cobre segundo a raça de ovinos, pois enquanto algumas têm menores capacidades de retenção do cobre em seus estoques orgânicos, outras o acumulam em demasia (Suttle, 2012). Também é reconhecido que ovinos jovens em crescimento são muito mais susceptíveis a desenvolverem quadros de intoxicação por cobre, pois podem absorver o cobre dietético de duas a três vezes mais eficientemente que os adultos (Suttle, 2010).

A patogenia da intoxicação cúprica acumulativa (ICA) em ovinos tem uma sequência determinada de eventos bioquímicos, apresentando duas fases distintas: pré-hemolítica e hemolítica. No decorrer de meses ou mesmo anos após a ingestão de dietas ricas em cobre, ocorre o acúmulo dele no organismo, principalmente nos hepatócitos periféricos à veia central e na zona pericanular. Quando os estoques hepáticos de cobre ultrapassam um determinado limite, geralmente superior a 1400ppm, pode ocorrer um rompimento dos lisossomos, provocando necrose intracelular. Esse processo pode resultar em morte celular de um número muito mais alto de hepatócitos, havendo uma súbita liberação do elemento livre cobre para o organismo, que, em pouco tempo, migra aos eritrócitos, acarretando intensa hemólise e severos danos renais, que constituem a principal causa mortis (Ferreira et al., 2008).

Desde a década de 50, sabe-se que o molibdênio, assim como o enxofre, interfere na disponibilidade do cobre para ruminantes. Estudos esclareceram que o molibdênio e o enxofre formam complexos chamados tiomolibdatos no rúmen, tornando o cobre altamente indisponível para o organismo (Gould e Kendall, 2011). Concluiu-se, dessa forma, que quanto maior a saturação da molécula de molibdato por enxofre, maior será o poder quelante do tiomolibdato sobre o cobre, tornando-o indisponível. Esses compostos estão mais presentes quanto maior o teor na dieta de fibra, de sulfato e de protozoários no conteúdo ruminal (Suttle, 1991).

Objetivou-se avaliar a potencialidade que um sal mineral rico em molibdênio $(300 \mathrm{ppm})$ pode ter na prevenção da ICA em ovinos. Para tal, comparou-se o metabolismo do cobre, do molibdênio e do zinco quanto a suas concentrações nos estoques séricos e hepáticos e o quadro clínico de ovinos jovens, que receberam, por um período relativamente prolongado, dietas com teores normais, altos ou muito altos de cobre.

\section{MATERIAL E MÉTODOS}

O presente estudo foi aprovado pela Comissão de Bioética (protocolo no 437/2004) da Faculdade de Medicina Veterinária e Zootecnia da Universidade de São Paulo.

Este trabalho foi conduzido na Faculdade de Medicina Veterinária e Zootecnia da Universidade de São Paulo, no campus de Pirassununga, São Paulo, Brasil (21 57' 29.61" S; 47²7'08.24" O).

Foram utilizados 25 ovinos machos, cruzados da raça Ile-de-France, hígidos, com idade em torno de três meses de idade e com cerca de $25 \mathrm{~kg}$ de peso vivo no início do experimento. Todos os animais foram vermifugados com antihelmínticos à base de ivermectina durante o período de adaptação, que durou um mês, com a finalidade de os ovinos se adaptarem à dieta e ao local de experimentação. Os animais foram mantidos em gaiolas metabólicas metálicas individuais, dispostas lado a lado a fim de diminuir o estresse dos animais.

Este experimento seguiu um delineamento experimental inteiramente ao acaso, em esquema multifatorial $(2 \times 2)$ (cobre dietético alto e altíssimo e suplementação mineral com e sem molibdênio), adicionado de um grupo controle com teores adequados de cobre na dieta $\mathrm{e}$ suplementado com sal mineral contendo molibdênio. Os 25 ovinos foram distribuídos aleatoriamente em cinco grupos, de cinco animais cada, e receberam os seguintes tratamentos dietéticos: 
grupo 1 - fornecimento de dieta composta de $80 \%$ da MS de feno de capim coast-cross (Cynodon dactlylum (L.) Pers), 20\% da MS de concentrado comercial e sal mineral ad libitum, contendo 600ppm de cobre quelatado e 300ppm de molibdênio;

grupo 2 - oferecimento de dieta composta de $50 \%$ da MS de feno de capim coast-cross (Cynodon dactlylum (L.) Pers), 50\% da MS de concentrado comercial e sal mineral ad libitum, contendo 600ppm de cobre quelatado, sem a presença de molibdênio; grupo 3 - dieta oferecida ao grupo 2, sendo adicionados à suplementação mineral 300ppm de molibdênio;

grupo 4 - dieta fornecida ao grupo 2, acrescida de $150 \mathrm{mg} /$ dia de sulfato de cobre penta-hidratado, misturado ao concentrado;

grupo 5 - dieta fornecida ao grupo 3 , acrescida de $150 \mathrm{mg} /$ dia de sulfato de cobre penta-hidratado, misturado ao concentrado.

Os teores de cobre e de molibdênio presentes no feno de capim coast-cross, no concentrado comercial e no sal mineral encontram-se na Tab. 1.

Tabela 1. Composição mineral das fontes de alimento e de suplementação mineral

\begin{tabular}{lcccc}
\multicolumn{1}{c}{ Micromineral } & $\begin{array}{c}\text { Feno de capim } \\
\text { coast-cross }\end{array}$ & $\begin{array}{c}\text { Concentrado } \\
\text { comercial }\end{array}$ & $\begin{array}{c}\text { Sal mineral com } \\
\text { molibdênio }\end{array}$ & $\begin{array}{c}\text { Sal mineral sem } \\
\text { molibdênio }\end{array}$ \\
\hline Cobre $(\mathrm{ppm})$ & 6,5 & 9,0 & 600 & 600 \\
Molibdênio $(\mathrm{ppm})$ & 1,1 & 0,6 & 300 & - \\
\hline
\end{tabular}

Fonte: Laboratório de Doenças Nutricionais e Metabólicas do Departamento de Clínica Médica da FMVZ/USP.

A quantidade total de MS oferecida aos animais foi a correspondente a $2,75 \%$ do peso corpóreo, corrigida quinzenalmente no decorrer do experimento.

A indução do quadro de intoxicação cúprica foi realizada mediante $\mathrm{o}$ fornecimento de alta quantidade de concentrado, associado ou não a $150 \mathrm{mg} /$ dia de sulfato de cobre penta-hidratado. Antes do fornecimento da ração concentrada aos animais, uma pequena quantidade desta era misturada com $150 \mathrm{mg}$ de sulfato de cobre pentahidratado e oferecida aos ovinos dos grupos $4 \mathrm{e}$ 5. Era aguardado seu consumo total antes de se fornecer o restante da alimentação. Caso algum animal recusasse o alimento com cobre, este era diluído em $20 \mathrm{~mL}$ de água e fornecido via oral com o auxílio de uma seringa de plástico.

Foram realizadas três séries de biópsias hepáticas durante o experimento, segundo técnica descrita por Minervino et al. (2009): a primeira durante o período de adaptação dos ovinos, a segunda após 75 dias do início do período experimental e a terceira ao término do experimento (no $150^{\circ}$ dia em ovinos que não manifestaram crise hemolítica, ou logo em seguida à manifestação clínica da ICA).
Foram realizados três ensaios de retenção do cobre e do molibdênio: um antes do início do experimento, outro na $10^{\mathrm{a}}$ semana e o último na $20^{\mathrm{a}}$ semana. Foi coletado em cada ensaio, no período de três dias, o total de fezes e de urina e quantificado o total de cobre e molibdênio excretados, além de se determinar o total desses microelementos ingeridos no decorrer desses dias, com pesagem do alimento ofertado e das sobras e desperdício, com a finalidade de se estabelecer o total desses microminerais retidos pelos ovinos.

As fezes foram coletadas por meio de bolsa coletora clássica, utilizada em ensaios de digestibilidade em ovinos, enquanto a urina foi recolhida em recipientes plásticos sob o funil coletor da base da gaiola metabólica. Nesse período, fezes eram coletadas e pesadas diariamente, e amostras eram congeladas, enquanto a urina era coletada e medida duas vezes ao dia, e as alíquotas eram congeladas.

A retenção aparente de cobre e de molibdênio no organismo foi obtida da diferença dos teores totais do micromineral ingerido menos o total excretado por dia. 
As amostras biológicas foram digeridas, e os teores de cobre, zinco e molibdênio determinados por meio de espectrofotometria de absorção atômica de acordo com protocolo descrito por Minervino (2011).

Os dados foram analisados quanto a sua distribuição normal pela prova de KolmogorovSmirnov. Posteriormente, esses dados foram avaliados segundo testes estatísticos paramétricos, por meio de teste $\mathrm{F}$ (análise de variância), e, quando significativas, as médias foram confrontadas pelo teste de Tukey-Kramer. A frequência de mortalidade dos grupos foi comparada pelo teste exato de Fisher (Massad et al., 2004). Foi adotado o nível de significância de $5 \%(\mathrm{P}<0,05)$ para todas as análises realizadas. O coeficiente de correlação foi avaliado mediante teste $\mathrm{F}$, ficando estabelecido que houve uma correlação de alta intensidade entre as variáveis quando $\mathrm{r}>0,60$; média intensidade quando $0,30<\mathrm{r}<0,60$; e baixa intensidade quando $r<0,30$, sendo o nível de significância obtido para todas as correlações igual ou inferior a 0,05 .

\section{RESULTADOS}

Nenhum ovino dos grupos 1, 2 e 3 apresentou qualquer alteração clínica ou manifestação de intoxicação cúprica acumulativa (ICA) durante o período experimental. Contudo, quatro ovinos dos grupos suplementados com cobre (4 e 5) manifestaram quadro clínico definitivo de ICA, sendo três carneiros do grupo 4 e um ovino do grupo 5. Houve o surgimento do quadro clínico de ICA nos três animais do grupo 4 nos $98^{\circ}, 126^{\circ}$ e $142^{\circ}$ dias , enquanto no ovino do grupo 5 ocorreu no $140^{\circ}$ dia. Ao se aplicar o teste exato de Fisher, não se constatou diferença entre a frequência de ocorrência de ICA entre os grupos 4 e $5(\mathrm{P}=0,52)$.

Todos os animais com ICA apresentaram quadro clínico considerado clássico, com presença de diminuição do apetite progressiva previamente ao surgimento de hemoglobinúria, apatia, andar cambaleante, diminuição dos movimentos ruminais, desidratação, mucosas aparentes e pele ictéricas, fezes pastosas e oligúria. Os ovinos acometidos de ICA também apresentaram perda de peso.

Em relação à retenção de cobre no organismo, dentro de cada grupo, constataram-se maiores quantidades nos animais dos grupos 4 e 5 na $10^{a}$ e $20^{\mathrm{a}}$ semanas quando comparadas com o período pré-experimental, e nos animais do grupo 3 na $20^{a}$ semana quando comparada com a semana zero. Na comparação entre grupos, observaram-se maiores retenções de cobre nos carneiros dos grupos 4 e 5 em relação aos grupos 1,2 e 3 tanto na $10^{\mathrm{a}}$ como na $20^{\mathrm{a}}$ semana (Tab. 2).

Tabela 2. Valores médios e desvios-padrão de cobre e de molibdênio retidos ( $\mathrm{mg}$ ) por dia em ovinos que receberam diferentes doses de cobre dietético

\begin{tabular}{ccccccc}
\hline & \multicolumn{5}{c}{ Grupos } & \\
& Semana & 1 & 2 & 3 & 4 & 5 \\
\hline Cobre & 0 & $-1,7 \pm 5,9$ & $-2,9 \pm 2,1 \mathrm{~B}$ & $-2,3 \pm 2,5$ & $-2,9 \pm 4,6 \mathrm{~B}$ & $-3,4 \pm 3,3 \mathrm{~B}$ \\
retido $(\mathrm{mg})$ & 10 & $-0,4 \pm 1,4 \mathrm{~b}$ & $1,9 \pm 4,3 \mathrm{bAB}$ & $0,0 \pm 2,8 \mathrm{~b}$ & $21,2 \pm 15,4 \mathrm{aA}$ & $18,8 \pm 12,2 \mathrm{aA}$ \\
& 20 & $-1,1 \pm 2,4 \mathrm{~b}$ & $2,4 \pm 2,4 \mathrm{bA}$ & $0,7 \pm 4,9 \mathrm{~b}$ & $25,9 \pm 12,2 \mathrm{aA}$ & $18,4 \pm 7,6 \mathrm{aA}$ \\
& & & & & & \\
Molibdênio & 0 & $0,9 \pm 0,8 \mathrm{~B}$ & $0,5 \pm 0,8$ & $0,6 \pm 0,5 \mathrm{~B}$ & $0,5 \pm 1,0$ & $0,3 \pm 1,0$ \\
retido (mg) & 10 & $4,0 \pm 1,1 \mathrm{aA}$ & $0,2 \pm 0,8 \mathrm{~b}$ & $3,4 \pm 2,4 \mathrm{aA}$ & $0,0 \pm 0,6 \mathrm{~b}$ & $1,8 \pm 0,8 \mathrm{ab}$ \\
& 20 & $4,2 \pm 0,6 \mathrm{aA}$ & $0,5 \pm 1,0 \mathrm{bc}$ & $3,1 \pm 1,8 \mathrm{abA}$ & $0,3 \pm 0,6 \mathrm{c}$ & $2,1 \pm 1,3 \mathrm{abc}$ \\
\hline
\end{tabular}

Nota: Letras minúsculas distintas nas linhas indicam diferenças significativas entre os grupos $(\mathrm{P}<0,05)$. Letras maiúsculas distintas nas colunas indicam diferenças significativas entre as semanas $(\mathrm{P}<0,05)$.

Já na retenção de molibdênio no organismo, dentro de cada grupo, constataram-se maiores quantidades nos ovinos dos grupos 1 e 3 na $10^{\mathrm{a}} \mathrm{e}$ $20^{\mathrm{a}}$ semanas quando comparadas com o período pré-experimental. Na comparação entre grupos, observaram-se maiores retenções de molibdênio nos carneiros dos grupos 1 e 3 em relação aos grupos 2 e 4 na $10^{\text {a }}$ semana, e os ovinos do grupo 1 apresentaram valores mais elevados do que os apresentados pelos grupos 2 e 4, assim como os animais do grupo 3 apresentaram maior retenção aparente de molibdênio que os animais do grupo 4 na $20^{a}$ semana (Tab. 2). 
A Tab. 3 mostra o aumento nos teores de cobre hepático nos grupos 2, 4 e 5, que atingiram seus valores mais altos na terceira biópsia; não houve diferença dentro dos grupos 1 e 3. Quando foram comparados os resultados obtidos entre os grupos, verificou-se que, já na segunda biópsia, os grupos 4 e 5 apresentavam valores superiores aos do grupo 1, na terceira biópsia, os grupo 4 e 5 continuavam a apresentar valores superiores aos grupos 1, 2 e 3, e os animais do grupo 2 mostravam resultados superiores aos do grupo 1 .

Tabela 3. Valores médios e desvios-padrão do cobre, do molibdênio e do zinco hepático (ppm) em ovinos que receberam diferentes doses de cobre dietético

\begin{tabular}{|c|c|c|c|c|}
\hline \multirow{2}{*}{ Mineral } & \multirow{2}{*}{ Grupo } & \multicolumn{3}{|c|}{ Dias } \\
\hline & & 0 & 75 & 150 \\
\hline \multirow{5}{*}{ Cobre } & 1 & $320 \pm 20$ & $318 \pm 53 b$ & $297 \pm 13 c$ \\
\hline & 2 & $339 \pm 27 B$ & $387 \pm 49 \mathrm{ABb}$ & $436 \pm 63 \mathrm{Ab}$ \\
\hline & 3 & $325 \pm 36$ & $329 \pm 22 b$ & $368 \pm 23 b c$ \\
\hline & 4 & $330 \pm 34 \mathrm{C}$ & $1490 \pm 254 \mathrm{Ba}$ & $3097 \pm 544 \mathrm{Aa}$ \\
\hline & 5 & $328 \pm 36 \mathrm{C}$ & $1413 \pm 253 \mathrm{Ba}$ & $2068 \pm 419$ Aa \\
\hline \multirow{5}{*}{ Molibdênio } & 1 & $3,7 \pm 0,9 \mathrm{~B}$ & $6,8 \pm 1,8 \mathrm{Aa}$ & $5,9 \pm 1,3 \mathrm{ABa}$ \\
\hline & 2 & $3,5 \pm 1,7$ & $3,5 \pm 1,8 b$ & $3,4 \pm 0,5 b$ \\
\hline & 3 & $3,6 \pm 0,9$ & $5,7 \pm 2,3 \mathrm{ab}$ & $5,4 \pm 1,5 \mathrm{ab}$ \\
\hline & 4 & $3,7 \pm 0,8$ & $3,9 \pm 1,1 \mathrm{~b}$ & $3,8 \pm 0,9 \mathrm{ab}$ \\
\hline & 5 & $3,6 \pm 1,1$ & $4,8 \pm 1,1 \mathrm{a}$ & $4,8 \pm 1,0 \mathrm{ab}$ \\
\hline \multirow{5}{*}{ Zinco } & 1 & $138 \pm 22$ & $139 \pm 11 b$ & $143 \pm 32 b$ \\
\hline & 2 & $127 \pm 31$ & $126 \pm 46 b$ & $140 \pm 28 b$ \\
\hline & 3 & $127 \pm 22$ & $115 \pm 21 b$ & $120 \pm 11 b$ \\
\hline & 4 & $130 \pm 15 B$ & $241 \pm 85 \mathrm{Aa}$ & $257 \pm 92 \mathrm{Aa}$ \\
\hline & 5 & $120 \pm 20 \mathrm{~B}$ & $217 \pm 57 \mathrm{Aa}$ & $259 \pm 96 \mathrm{Aa}$ \\
\hline
\end{tabular}

Nota: Letras maiúsculas distintas nas linhas indicam diferenças significativas entre os tempos para o mesmo micromineral $(\mathrm{P}<0,05)$. Letras minúsculas distintas nas colunas para o mesmo micromineral indicam diferenças significativas entre os grupos $(\mathrm{P}<0,05)$.

Em relação aos valores médios da concentração hepática de molibdênio, pode-se ressaltar o aumento nos teores de molibdênio no grupo 1, que atingiu seus valores mais altos na segunda biópsia; dentro dos grupos 2, 3, 4 e 5, não houve diferença. Quando foram comparados os resultados obtidos entre os grupos, verificou-se que, já na segunda biópsia, os grupos 1 e 5 apresentavam valores superiores aos dos grupos 2 e 4, e na terceira biópsia, o grupo 1 continuava a apresentar valores superiores aos do grupo 2, conforme demonstrado na Tab. 3.

Os teores de zinco hepático elevaram-se nos grupos 4 e 5 , que atingiram seus valores mais altos já na segunda biópsia; não houve diferença dentro dos grupos 1, 2 e 3. Quando foram comparados os resultados obtidos entre os grupos, verificou-se que, já na segunda biópsia, os grupos 4 e 5 apresentavam valores superiores aos dos grupos 1, 2 e 3, assim como na terceira biópsia, conforme apresentado na Tab. 3 .
A análise da relação entre o cobre hepático e a concentração de zinco hepático mostrou uma alta correlação positiva $(r=0,74)$. Foi também constatada uma significativa correlação negativa entre a ingestão de molibdênio e os teores de cobre hepático $(r=-0,72)$. Como era de se esperar, a retenção diária de cobre pelo organismo refletiu, de maneira muita significativa, os teores de cobre hepático nos animais $\left(\mathrm{r}=0,83 ; \mathrm{R}^{2}=0,69\right)$, confirmando o conceito clássico de que o fígado é o grande órgão-estoque de cobre nos ovinos.

\section{DISCUSSÃO}

O uso metafilático contra ICA de sal mineral contendo molibdênio deve ser interpretado com muita atenção neste estudo. Numericamente, poder-se-ia dizer que esse sal contribui para uma menor incidência de ICA nos ovinos no grupo 5 (20\%; $1 / 5)$ em relação ao grupo $4(60 \% ; 3 / 5)$, que atuou como controle sem molibdênio, ambos 
altamente expostos à expressiva ingestão de cobre. Entretanto, quando aplicado o teste exato de Fisher, não houve diferença $(\mathrm{P}=0,52)$ para incidência de ICA entre os grupos. Deve-se ressaltar que, devido ao pequeno número de graus de liberdade, esse teste é altamente rigoroso e apenas seria significativo caso houvesse uma diferença de, no mínimo, quatro ovinos acometidos entre os grupos 5 e 4 . Provavelmente, se o período experimental fosse estendido em mais cinco semanas, seria possível, uma vez que os animais restantes do grupo 4 já estavam apresentando hiporexia. Esse prolongamento do período experimental também foi verificado por Pereira (2008), que necessitou de uma média de 30 semanas para intoxicar experimentalmente ovinos com cobre.

Os sinais clínicos apresentados pelos animais que manifestaram ICA são compatíveis com os descritos na literatura (Ferreira et al., 2008; Miguel et al., 2013). A perda de peso progressiva dos animais com ICA provavelmente foi decorrente da hiporexia progressiva e/ou da anorexia apresentadas.

A maior presença do molibdênio na dieta, entretanto, esteve diretamente relacionada com um menor acúmulo de cobre, comprovado pelo coeficiente de correlação negativo $(r=-0,72)$. Uma menor retenção de cobre no fígado pode sugerir o efeito preventivo para ocorrência de ICA, promovida pela adição do molibdênio na dieta na forma de sal mineral.

Era de se esperar que a suplementação com sulfato de cobre aumentasse as reservas de cobre nos animais, visto que a disponibilidade desse elemento nesse composto é alta, próxima a 50\% (Suttle, 2010). Essa dieta provocou acúmulo de cobre hepático, aumentando em quase $30 \%$ os teores de cobre ao fim de 150 dias.

Várias condições poderiam explicar esse acúmulo de cobre no fígado. Embora os teores de cobre na ração concentrada (9ppm) fossem ligeiramente mais elevados que na forragem (6,5ppm), a disponibilidade do cobre nos concentrados é cerca de três a cinco vezes maior que nos capins, além de normalmente apresentar teores baixos de molibdênio (Ortolani, 2006; Suttle, 2010).
Suttle (2012) encontrou menores teores de cobre em animais suplementados com molibdênio, enxofre e zinco. Já Sinclair (2013) verificou que, em vacas de leite, a adição de molibdênio e enxofre na dieta resulta em menor acúmulo de cobre hepático. Conti (2014) afirmou que a adição de molibdênio reduz os níveis de cobre.

Um fato interessante ocorreu com os teores de zinco hepático nos animais dos grupos submetidos à grande suplementação de cobre (4 e 5), já que estes apresentaram um acúmulo muito maior de zinco do que os demais grupos (Tab. 1). Observou-se ainda que quanto mais altos os teores de cobre hepático, maiores os de zinco no fígado. Idêntico resultado foi obtido por Minervino et al. (2009b), que intoxicaram experimentalmente bovinos e búfalos com cobre, e Minervino (2011), em ovinos.

O zinco é o principal estimulador da síntese de metalotioneína hepática. Segundo López-Alonso et al. (2005), quanto maior a concentração de zinco no fígado, maior é o teor de metalotioneína nesse órgão $\left(\mathrm{R}^{2}=0,69\right)$. Esse composto é responsável pela complexação com o cobre estocado no órgão para ser posteriormente excretado pela bile no processo de desintoxicação. Embora não tenha determinado os teores de metalotioneína, Minervino (2007) sugeriu que ruminantes prestes a se intoxicarem por cobre passam a acumular zinco no fígado no afã de aumentarem a desintoxicação do cobre.

Os ovinos de todos os grupos receberam quantidades superiores à mínima recomendada (40ppm) de zinco na dieta (entre 55 e $75 \mathrm{mg}$ /ovino/dia). Mas ficou comprovado que os animais que receberam quantidades muito elevadas de cobre já passaram a concentrar maiores quantidades de zinco no fígado na $10^{\mathrm{a}}$ semana. Esse fenômeno pode ser interpretado como uma reação de alarme dos ovinos para se protegerem contra o excesso de cobre nos hepatócitos, por meio de maior produção de metalotioneína no órgão, apesar de os ovinos produzirem menor quantidade de metalotioneína hepática (Bremner, 1987). Segundo Minervino (2011), quanto maior o acúmulo de zinco hepático, maior o percentual de metalotioneína ligada ao cobre $\left(\mathrm{R}^{2}=0,46\right)$, e consequentemente maior sua excreção. 
Contudo, mesmo com um provável aumento na produção de metalotioneínas e também pelo maior percentual de sua ligação com o cobre, a quantidade de cobre acumulada no fígado dos ovinos foi muito elevada nos animais dos grupos 4 e 5 , desencadeando o quadro de ICA em alguns ovinos.

\section{CONCLUSÕES}

O aumento de concentrado na dieta provocou maior retenção de cobre no fígado dos ovinos. Não foi possível comprovar a eficiência do sal mineral rico em molibdênio em prevenir a ICA em ovinos que receberam dietas muito ricas em cobre, apesar de que quanto maior a ingestão de molibdênio na dieta, menor foi o acúmulo de cobre hepático ao término do experimento. Quanto mais altos os teores de cobre no fígado, maior foi a concentração de zinco nesse órgão.

\section{AGRADECIMENTOS}

Os autores agradecem à Fundação de Amparo à Pesquisa do Estado de São Paulo - Fapesp pelas concessões da bolsa de doutorado (processo $\mathrm{n}^{\circ}$ 03/09991-8) e do auxílio esquisa (processo $n^{\circ}$ 05/02291-6).

\section{REFERÊNCIAS}

BREMNER, I. Involvement of metallothionein in hepatic metabolism of copper - critical review. $J$. Nutr., v.117, p.19-29, 1987.

CONTI, R.M.C. Efeito de fontes orgânicas de cobre e enxofre sobre a interação cobre, molibdênio e enxofre na alimentação de ovinos. 2014. 84f. Tese (Doutorado) - Faculdade de Zootecnia e Engenharia de Alimentos, Universidade de São Paulo, Pirassununga, SP.

FERREIRA, M.B.; ANTONELLI, A.C.; ORTOLANI, E.L. Intoxicação por cobre, selênio, zinco e cloreto de sódio. In: SPINOSA, H.S.; GÓRNIAK, S.L.; PALERMO-NETO, J. Toxicologia aplicada à medicina veterinária. Barueri: Manole, 2008. p.665697.

GOULD, L.; KENDALL, N.R. Role of the rumen in copper and thiomolybdate absorption. Nutr. Res. Rev., v.24, p.176-182, 2011.

LÓPEZ-ALONSO, M.; PRIETO, F.; MIRANDA, M. et al. The role of metallothionein and zinc in hepatic copper accumulation in cattle. Vet. J., v.169, p.262267, 2005.
MASSAD, E.; MENEZES, R.X.; SILVEIRA, P.S.P. et al. Métodos quantitativos em medicina. Barueri: Manole, 2004. 561p.

MIGUEL, M.P.; SOUZA, M.A.; CUNHA, P.H.J. et $a l$. Intoxicação crônica por cobre em ovinos: conduta para o diagnóstico conclusivo. Arq. Bras. Med. Vet. Zootec., v.65, p.364-368, 2013.

MINERVINO, A.H.H. Estudo comparativo da susceptibilidade de bovinos e bufalinos à intoxicação cúprica acumulativa. 2007. 99f. Dissertação (Mestrado em Medicina Veterinária) - Faculdade de Medicina Veterinária e Zootecnia, Universidade de São Paulo, São Paulo, SP.

MINERVINO, A.H.H.; BARRÊTO JÚNIOR, R.A.; RODRIGUES, F.A.M.L. et al. Biópsia hepática por laparotomia paracostal em bovinos e búfalos. Ciênc. Rural, v.39, p.798-802, 2009a.

MINERVINO, A.H.H.; BARRÊTO JÚNIOR, R.A.; FERREIRA, R.N.F. et al. Clinical observations of cattle and buffalos with experimentally induced chronic copper poisoning. Res. Vet. Sci., v.87, p.473$478,2009 b$.

MINERVINO, A.H.H. Avaliação de crescentes teores de zinco dietético no metabolismo de cobre e na prevenção de intoxicação cúprica em ovinos. 2011. 69f. Tese (Doutorado em Medicina Veterinária) Faculdade de Medicina Veterinária e Zootecnia, Universidade de São Paulo, São Paulo, SP.

ORTOLANI, E.L. Macro e microelementos. In: SPINOSA, H. S.; GÓRNIAK, S. L.; BERNARDI, M. M. Farmacologia aplicada à medicina veterinária. 4.ed. Rio de Janeiro: Guanabara Koogan, 2006. p.750761.

PEREIRA, W.A.B. Intoxicação cúprica experimental em ovinos: aspectos clínicos e laboratoriais. 2008. 59f. Tese (Doutorado em Medicina Veterinária) Universidade Estadual Paulista, Jaboticabal, SP.

SINCLAIR, L.A.; HART, K.J.; JOHNSON, D. et al. Effect of inorganic or organic copper fed without or with added sulfur and molybdenum on the performance, indicators of copper status, and hepatic mRNA in dairy cows. J. Dairy Sci., v.96, p.43554367, 2013.

SUTTLE, N.F. The interaction between copper, molybdenum and sulphur in ruminant nutrition. Annu. Rev. Nutr., v.11, p.121-140, 1991.

SUTTLE, N.F. Mineral nutrition of lvestock. 4.ed. Oxforshire: CABI, 2010. 587p.

SUTTLE, N.F. Control of hepatic copper retention in Texel ram lambs by dietary supplementation with copper antagonists followed by a copper depletion regimen. Anim. Feed Sci. Technol., v.173, p.194-200, 2012. 